\title{
Scaling Relations of Dwarf Galaxies without Supernova-Driven Winds
}

\author{
K. Tassis ${ }^{1,2}$, A. V. Kravtsov ${ }^{1,2,3}$ \\ and N. Y. Gnedin ${ }^{1,4}$ \\ ${ }^{1}$ Department of Astronomy and Astrophysics, The University of Chicago, Chicago, IL 60637, \\ USA \\ ${ }^{2}$ The Kavli Institute for Cosmological Physics, The University of Chicago, Chicago, IL 60637, \\ USA \\ ${ }^{3}$ Enrico Fermi Institute, The University of Chicago, Chicago, IL 60637, USA \\ ${ }^{4}$ Particle Astrophysics Group, Fermilab, Batavia, IL 60510, USA
}

\begin{abstract}
Nearby dwarf galaxies exhibit tight correlations between their global stellar and dynamical properties, such as circular velocity, mass-to-light ratio, stellar mass, surface brightness, and metallicity. Such correlations have often been attributed to gas or metal-rich outflows driven by supernova energy feedback to the interstellar medium. We use high-resolution cosmological simulations of high-redshift galaxies with and without energy feedback, as well as analytic modeling, to investigate whether the observed correlations can arise without supernova-driven outflows. We find that the simulated dwarf galaxies exhibit correlations similar to those observed as early as $z \approx 10$ and the addition of realistic levels of supernova energy feedback has no appreciable effect on these correlations. We also show that the correlations can be well reproduced by our analytic model that accounts for gas inflow but without outflows, and star formation rate obeying the Kennicutt-Schmidt law with a critical density threshold. We argue that correlations in simulated galaxies arise due to the increasingly inefficient conversion of gas into stars in low-mass dwarf galaxies rather than supernova-driven outflows.
\end{abstract}

Keywords. stars: formation, galaxies: formation, cosmology: theory

\section{Introduction}

Observational studies of galaxies have revealed the existence of tight correlations between their global stellar and dynamical properties, such as circular velocity, mass-to-light ratio, stellar mass, surface brightness, and metallicity. For example, scalings of the mean metallicity of a galaxy with other global properties such as its luminosity, stellar mass, or total mass, have been established for galaxies of a large range of masses and morphologies (e.g., Lequeux et al. (1979), Garnett \& Shields (1987), Zaritsky et al. (1994), Garnett (2002), Prada \& Burkert (2002), Dekel \& Woo (2003), Tremonti et al. (2004), Pilyugin et al. (2004), Simon et al. (2006), Lee et al. (2006a)). Theoretical modelling of these correlations can help us to identify key physical processes shaping global properties of galaxies.

Supernova energy feedback to the interstellar medium (ISM) with putative associated gas outflows from galaxies has long been a favored mechanism to explain these correlations and other properties of low-mass galaxies Larson (1974), Dekel \& Silk (1986), Arimoto \& Yoshii (1987). The strong effect of the SN feedback on properties of low-mass galaxies is also a standard assumption of the semi-analytic models of galaxy formation Lacey et al. (1993), Kauffmann et al. (1993), Cole et al. (1994), Somerville \& Primack (1999), Benson et al. (2003), Croton et al. (2006). Dekel \& Woo (2003) argue, for example, that the correlations between circular velocity, stellar mass, metallicity, and stellar 
surface densities they find for the nearby dwarf galaxies can be reproduced with a simple semi-analytic model incorporating the effect of supernova feedback on the gas component of dwarf galaxies.

The existence of galactic winds has indeed been observationally established in starforming galaxies at low and high redshifts Veilleux et al. (2005), Heckman et al. (2000), Pettini et al. (2001), Martin et al. (2002), Strickland et al. (2004), Martin (2005), Ott et al. (2005). An indirect evidence for metal loss in winds is the high-metallicity of the diffuse intergalactic medium in groups and clusters Renzini et al. (1993). However, the extent to which the outflows affect the global properties of galaxies and whether the outflow gas escapes the gravitational potential of its host halo or rains back down to the disk remains uncertain. Mac Low \& Ferrara (1999) and D'Ercole \& Brighenti (1999) used numerical simulations to show that global gas blowout is inefficient in all but the smallest mass halos Ferrara \& Tolstoy (2000), Marcolini et al. (2006), although metal-enriched SN ejecta may be removed efficiently. It is also uncertain whether correlations of metallicity with galaxy stellar mass and mass-to-light ratios can be attributed solely to such winds.

In the context of cosmological simulations, the SN feedback is usually ineffective, unless ad hoc phenomenological recipes enhancing SN feedback are employed Navarro \& White (1993), Navarro \& Steinmetz (1997), Thacker \& Couchman (2001), Marri \& White (2003), Springel \& Hernquist (2003), Scannapieco et al. (2006). It is likely that this inefficiency is at least partially due to the inability of current cosmological simulations to resolve the scales and processes relevant to stellar feedback. However, it is also possible that the actual effects of feedback on the global properties of galaxies are fairly small in reality. Cosmological simulations at this point cannot make ab initio predictions about the importance or inefficiency of stellar feedback. Observations, on the other hand, although showing evidence for the presence of large-scale winds in actively starforming galaxies Veilleux et al. (2005), are rather uncertain in their estimates of wind mass loss to provide reliable direct observationally-motivated feedback recipes for implementation in simulations. Nevertheless, we can gauge t he importance of supernova energy injection by comparing galaxies formed in simulations performed with different assumptions about feedback to observations. In this respect, the smallest dwarf galaxies present the ideal test case because they can be expected to be the most susceptible to the effects of SN feedback due to their shallow potential wells Larson (1974), Dekel \& Silk (1986).

The role of feedback in the formation of dwarf galaxies has been investigated in several recent studies. Tassis et al. (2003) used Eulerian AMR simulations of galaxy formation with star formation and SN feedback and found that correlations between mass-to-light ratio and metallicity and stellar mass and metallicity, similar to those observed for the nearby dwarfs, are exhibited by dwarf galaxies in their simulations at $z \gtrsim 3$. The simulations of Tassis et al. (2003) used a rather extreme amount of energy per supernovae to maximize the effects of feedback. They did not test, however, whether feedback or some other mechanism is in fact the dominant factor in shaping the correlations. More recently, Kobayashi et al. (2006) found a tight relation between stellar metallicity and stellar mass at all redshifts in their Smooth Particle Hydrodynamics (SPH) cosmological galaxy formation simulations, which they attributed to the mass-dependent galactic winds. The winds in the model of Kobayashi et al. (2006) do affect the gas and metal content of galaxies significantly, with the effect increasing towards lower mass systems (see their Fig. 16), which plays an important role in shaping the resulting correlation of metallicity and stellar mass.

De Rossi et al. (2006) have recently studied the origin of luminosity- and stellar massmetallicity relations in cosmological simulations. They find that correlations similar to those observed arise already at high redshifts, although supernova feedback in their 
simulations is not efficient. This is consistent with our results presented below. Simon et al. (2006) seeked observational correlations between metallicity, surface brightness and mass-to-light ratio within a single galaxy (M33) and found that indeed such correlations exist. They used SPH simulations and analytical calculations to show that feedback is not responsible for these correlations. They found that the presence of feedback decreases the scatter in such relations, although this may at least in part be due to their numerical implementation of feedback. Most recently, Brooks et al. (2006) presented a study of the origin and evolution of the mass-metallicity relation in cosmological simulations. They also concluded that the relation arises not due to the mass loss in winds, but due to increasing inefficiency of star formation in smaller mass galaxies. However, they argue that low effective yields of dwarf galaxies can only be explained by the loss of metals in winds. We argue below that there is an alternative explanation for the low effective yields of dwarfs.

\section{Analytical Model for the Evolution of the Baryonic Component of Dwarf Galaxies}

In this section we present a simple analytic model which we use to follow the evolution of the baryonic component (gas, stars and metals) of dwarf galaxies at high redshifts. In following sections, we will make detailed comparisons of this model with our simulation results.

Our analytic model entails the following assumptions:

(1) Galaxies accrete gas at a rate consistent with simulations, but no gas or metals escape outside the virial radius of the parent halo.

(2) The gas mass of each galaxy is proportional to its total (dynamical) mass, $M_{\text {gas }} \propto$ $M$. At early epochs $M_{*} \ll M_{\text {gas }}$, which implies for the baryonic mass $M_{\mathrm{b}}=M_{\mathrm{gas}}+M_{*} \approx$ $M_{\text {gas }}$. The gas fraction within halos in our simulations is approximately independent of the total (dynamical) mass for $M_{\text {tot }} \gtrsim 10^{9} \mathrm{M}_{\odot}$. This is consistent with the results of independent simulations of Tassis et al. (2003). Therefore this assumption is an excellent approximation for all but the smallest objects in the simulation, which are excluded in our analysis of other global quantities. Although at lower redshifts the total baryon fraction in small mass galaxies $M<10^{10} M_{\odot}$ can be suppressed due to heating by the cosmic UV background Gnedin (2000), Hoeft et al. (2006), our results would still be applicable to galaxies in which a significant fraction of stars is formed at high redshifts before such suppression has occurred in the mass range we consider. We should stress that we intend to apply our model only to such stellar populations.

(3) The gas in galaxies forms an exponential disk with radial extent proportional to the virial radius of the parent halo. The disk is assumed to be more extended and consequently less compressed for smaller objects with $T_{\mathrm{vir}}<10^{4} \mathrm{~K}$, in which gas cannot cool efficiently.

(4) Gas is converted into stars at a rate given by the Kennicutt law of star formation with a density threshold Martin \& Kennicutt (2001). Gas at densities below the threshold does not form stars. Note that the Kennicutt law with the threshold is reproduced by our numerical simulations, but it is not assumed a priori. The numerical simulations implement a much simpler recipe for star formation.

(5) Instantaneous recycling approximation: i.e., no time delay is assumed between the birth of a generation of stars and the corresponding metal enrichment of the ISM. Hence, we implicitly assume that the metal enrichment due to type II supernovae is dominant. This is appropriate for all but the most massive dwarf galaxies. 
The key ingredient of our model is the relation between the gas mass and the stellar mass of a galaxy. We will first derive this correlation, and subsequently use it to derive relations between the stellar mass, gas mass, stellar surface density, metallicity, and circular velocity of high- $z$ galaxies.

\subsection{Stellar Mass - Gas Mass Relation}

The relation between stellar mass and total gas mass in dwarf galaxies is central to understanding the origin of correlations between other observable quantitiest. In this section, we derive such a relation. In subsequent sections, we will use it to explain the observed correlations and we will directly compare it against our simulation results.

The rate of change of the gas mass, assuming that there are no significant gas outflows due to $\mathrm{SN}$-driven winds, is

$$
\frac{d}{d t}\left(M_{\text {gas }}\right)=F-\psi
$$

where $F$ is the gas mass accretion rate and and $\psi$ is the star formation rate. The rate of change of the stellar mass is

$$
\frac{d M_{*}}{d t}=\psi .
$$

If the dependence of $\psi$ and $F$ on $M_{\text {gas }}$, as well as any explicit dependence on redshift, are known, then equations (2.1) and (2.2) can be integrated in time as a system of two ordinary differential equations. Different initial conditions for $M_{\text {gas }}$ at some early time (for which we can safely assume that $\left.M_{*}=0\right)$ will produce different pairs of $\left(M_{\mathrm{gas}}, M_{*}\right)$ at any time of interest. In this way, we calculate parametrically the prediction of our analytic model for the $M_{*}-M_{\text {gas }}$ relation. $F$ is proportional to the gas mass and $\psi$ depends on the extent of the gaseous disk (which in turn depends on the dynamical mass of the galaxy and the angular momentum parameter, $\lambda$ ) and the star formation density threshold, $\Sigma_{\mathrm{th}}$. The functional dependence of $F$ and $\psi$ on the gas mass is presented in detail in Tassis et al. (2007).

The free parameters which control the normalization and the shape of the $M_{\mathrm{gas}}-M_{*}$ relation are $\lambda$ and $\Sigma_{\mathrm{th}}$.

Remarkably, we find that the best fit to our data at $z=4$ is obtained when both free parameters have their fiducial values, $\lambda=0.033$ (the most probable value) and $\Sigma_{\mathrm{th}}=5 \mathrm{M}_{\odot} \mathrm{pc}^{-2}$ (the "canonical" observational value).

\subsection{Mass-to-light ratio vs. metallicity}

Under the assumptions of negligible inflow or outflow of metals and instantaneous recycling, and for a fixed initial mass function, the mass of metals of low- $Z$, high- $z$ galaxies is proportional to the stellar mass $\ddagger$. From $M_{\mathrm{Z}}=Z M_{\mathrm{gas}} \propto M_{*}$ we can immediately write

$$
Z \propto \frac{M_{*}}{M_{\mathrm{gas}}} .
$$

$\dagger$ This relation may not hold for some galaxies, if, for instance, their gas is lost due to tidal or ram pressure stripping.

$\ddagger$ For this proportionality to hold, $Z$ needs to be low enough so that the mass of freshly produced metals is $\gg$ than the mass of metals lost to long-lived stars and stellar remnants, which can be shown to be true for $Z \ll 0.02$ for the metallicity enhancement scheme implemented in our simulations, and which always holds in our simulated galaxies. 
Assumption (2), $M_{\text {gas }} \propto M$, then gives $Z \propto M_{*} / M$. The luminosity of an object, $L$, can be considered proportional to its stellar mass $\Phi$. Then, Eq. (2.3) becomes $Z \propto L / M$ or

$$
M / L \propto Z^{-1},
$$

in agreement with observations and, as shown in $\S 3$, the results of our simulations. This is a simple interpretation of the correlation between the mass-to-light ratio and metallicity found by Prada \& Burkert (2002).

\subsection{Additional Correlations}

We can use the $M_{\text {gas }}-M_{*}$ relation we derived in $\S 2.1$ in combination with Eq. (2.3) and assumptions $M_{\text {gas }} \propto M$ and $L \propto M_{*}$ to derive additional relations between stellar mass and stellar surface density, metallicity, and circular velocity of high- $z$ galaxies. We will overplot these relations with our simulation results and discuss them in $\S 3$.

\section{Results}

In this section, we discuss relations between global properties of galaxies in our cosmological simulations, how these depend on the detailed physics included in each simulation, and how they compare to the observationally established correlations and our analytic models of $\S 2$.

The panels in the figures discussed in this section are arranged in the following way: left panel - FEC simulation (SN feedback, uniform UV background, tabulated heating/cooling rates); middle panel - NFEC simulation (no SN energy feedback, uniform UV background, tabulated heating/cooling rates); right panel - FNEC-RT simulation (SN feedback, 3-D radiative transfer). We do not show the results of the F2NEC-RT simulations in all of the figures for clarity. In all cases, the results of the F2NEC-RT simulations are quite similar to those of the FNEC-RT run.

\subsection{Correlations Involving Metallicities}

Before we proceed to discuss the correlation of the metallicities in the simulated galaxies with other observables, we should make a note about the distinction between stellar and gas metallicities. Metallicities in stars represent an average metallicity over the star formation history of the observed object, while gas metallicities are actually representative of the amount of metals currently present in the interstellar medium. Observationally, either one or both can be measured and used. For example, Dekel \& Woo (2003) use stellar metallicities for $\mathrm{dE}$ galaxies and gas oxygen abundance for dIrr, while Prada \& Burkert (2002) use stellar metallicities in all cases. The metallicity in the gas and in the stars, although not the same in general, are related to each other. This is particularly true for the high-redshift galaxies in our simulations. Figure 1 shows the metallicity in the gas versus the metallicity in stars for every object in our simulations at different redshifts. The solid line corresponds to the one-to-one relation $Z_{\text {gas }}=Z_{*}$. Indeed, for the objects and redshifts we consider, the metallicity of stars tracks that of the gas very closely.

A tight correlation observed for the nearby dwarf galaxies is the correlation of the dynamical mass-to-light ratio and metallicity Prada \& Burkert (2002). In $\S 2.2$ we showed that such a correlation should naturally arise in low-metallicity dwarf galaxies without gas outflows and regardless of their star formation rate as long as their baryon mass is

I This approximation is appropriate for older stellar populations. We use it here because we assume that the stellar populations in our high- $z$ galaxies would actually be observed today, when they have aged substantially. 


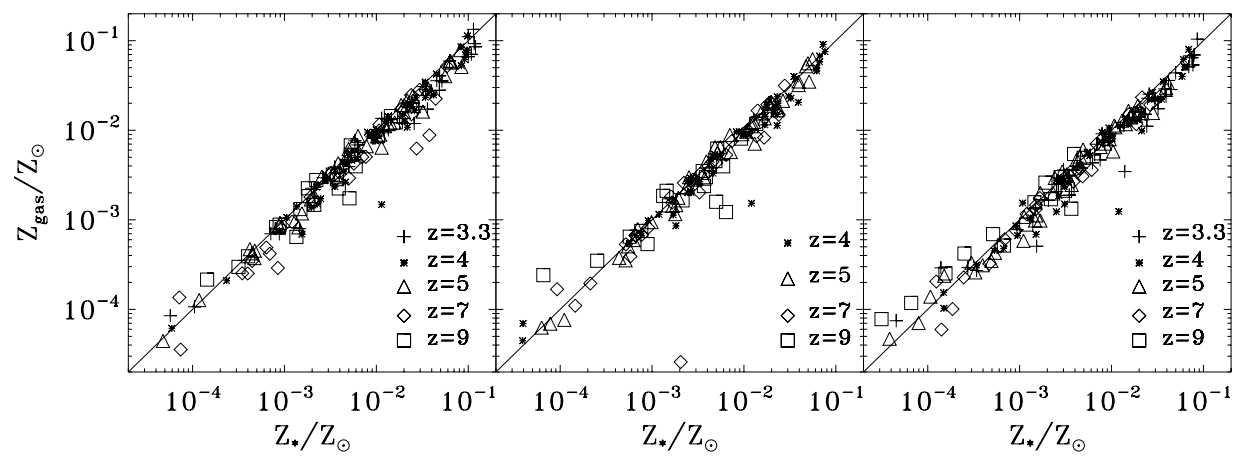

Figure 1. Gas metallicity vs. stellar metallicity. Left panel: simulation FEC; middle panel: simulation NFEC; right panel: FNEC-RT simulation. Different symbols correspond to different redshifts as detailed in the legend. The solid line corresponds to $Z_{\mathrm{gas}}=Z_{*}$.

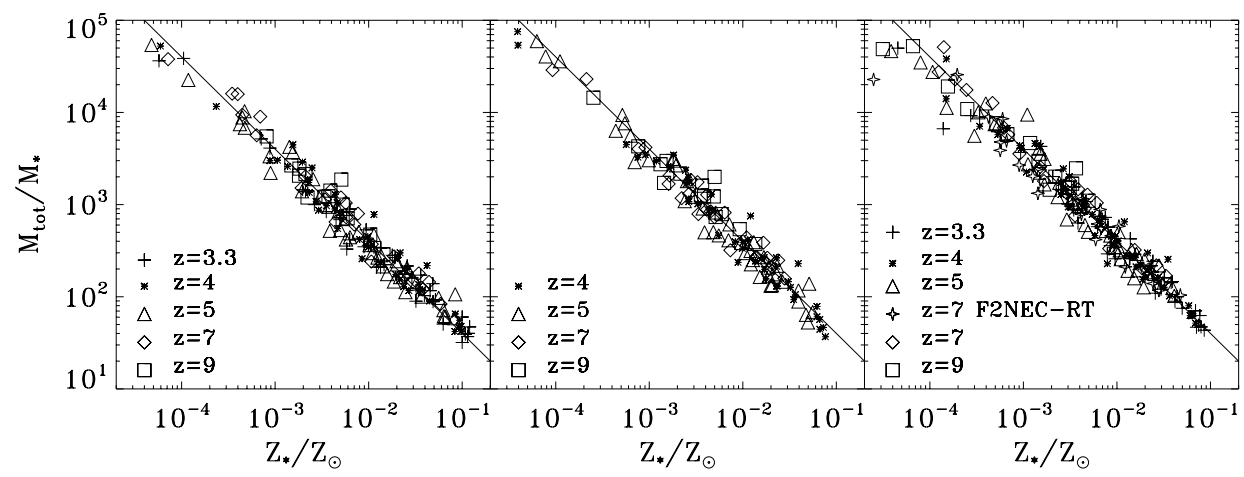

Figure 2. Ratio between total mass and stellar mass (assumed to be proportional to the massto-light ratio of each object), plotted against stellar metallicity. Left panel: simulation FEC; middle panel: simulation NFEC; right panel: FNEC-RT simulation. Different symbols correspond to different redshifts as detailed in the legend. The solid line corresponds to the $M_{\text {tot }} / M_{*} \propto Z^{-1}$ scaling

approximately proportional to their total mass. Figure 2 clearly shows that for galaxies in our simulations the ratio of the total (baryonic + dark matter) to stellar mass is tightly correlated with metallicity, independently of the details of star formation and cooling and inclusion of SN feedback. The form of the correlation, $M / M_{*} \propto Z^{-1}$ is similar to that exhibited by the observed dwarf galaxies. The correlation arises for objects at the earliest epochs, which implies that a similar correlation should be expected to hold for the observed galaxies at high redshifts.

Basic assumptions in the derivation of this correlation is that $M_{\text {gas }} \propto M$ and $Z \ll Z_{\odot}$, implying that at the time this correlation is being established, the galaxy is in the gas-rich, low-metallicity regime. For galaxies in which star formation is inefficient and which have not lost substantial amount of gas (due to, e.g., ram or tidal stripping), it is clear that both assumptions are likely to hold until late times. Such galaxies may move along the correlation as they evolve, but they will remain on the same line. However, such galaxies are not the only objects which may retain this correlation until the current cosmic epoch: in galaxies where star formation is halted early on (when our derivation assumptions are still valid) and gas is stripped from them, neither the metallicity nor the dark matter or 


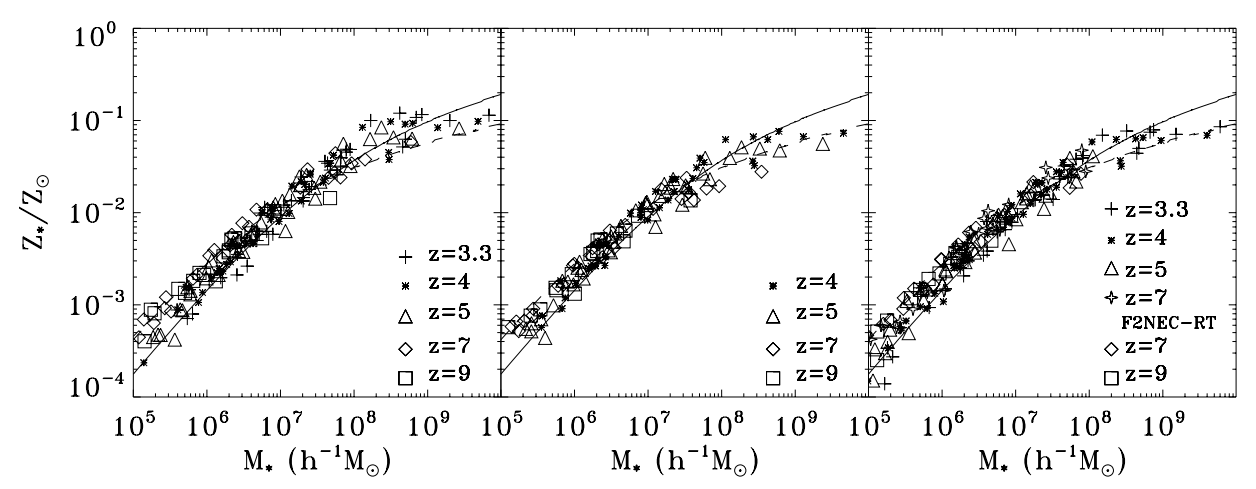

Figure 3. Stellar metallicity as a function of stellar mass. Left panel: simulation FEC; middle panel: simulation NFEC; right panel: FNEC-RT simulation. Different symbols correspond to different redshifts as detailed in the legend. The solid and dashed lines show the results of our analytic model for $z=4$ and $z=9$, respectively.

the stellar mass of the galaxy would be affected. Hence the correlation between $M / M_{*}$ and $Z$ would be preserved. This would then be a typical example of a "fossil" correlation, established in the early phases of the evolution of a galaxy and preserved until the present cosmic epoch due to lack of additional evolution of the observable quantities involved.

Figure 3 shows the mass-weighted metallicity of stars of the simulated galaxies as a function of stellar mass. The correlation of the two quantities is very tight and can be described by a power law at small masses. At large stellar masses, $M_{*} \gtrsim 5 \times 10^{8} \mathrm{M}_{\odot}$, the correlation becomes shallower. This is qualitatively consistent with the flattening of the $Z-M_{*}$ relation found in the SDSS data Tremonti et al. (2004), although in their observations the break occurs at much larger masses: $m \sim 3 \times 10^{10} \mathrm{M}_{\odot}$. Note, however, that this figure should not be directly compared to the present-day correlation over a wide range of stellar masses because massive galaxies will undergo substantial evolution in both their stellar mass and metallicity between $z=3$ and the present, while the evolution of dwarf galaxies is expected to be much slower. For some of the dwarf galaxies the evolution can be halted at high redshifts if their gas is removed by some process (e.g., ram pressure stripping). Thus, the comparison with the correlations exhibited by the local galaxies is most meaningful for smaller mass objects.

The solid lines in Figure 3 show the results of our analytic model. The model reproduces the slope of the correlation in the two mass regimes, the location of the break, and the observed redshift evolution seen in the simulated galaxies. The correlation in the model arises mainly due to the inefficiency of star formation in dwarf galaxies and the existence of star formation threshold, without gas outflows. Interestingly, direct extrapolation of the model to $z=0$ indicates that the flattening of the $M_{*}-Z$ relation shifts to $M_{*} \sim$ few $\times 10^{10} \mathrm{M}_{\odot}$, in agreement with the SDSS data. However, the reader should keep in mind that certain assumptions $\left(Z \ll Z_{\odot}, M_{*} \ll M_{\text {gas }}\right)$ of our analytic model are expected to eventually break down at late times (especially for the higher-mass objects), and hence additional effects may become important in shaping the present-day properties of higher-mass galaxies.

\section{Conclusions}

In this paper, we use a suite of high-resolution cosmological simulations to examine the role of different physical processes in establishing the observed scaling relations of dwarf 
galaxies. All simulations included a recipe for star formation and metal enrichment of the ISM. Three simulations (FEC, FNEC-RT, F2NEC-RT) additionally included energy feedback from supernovae to the ISM. Simulations FNEC-RT and F2NEC-RT followed detailed 3-D radiative transfer from individual UV sources and the chemistry network of ionic species of hydrogen, helium, and molecular hydrogen to determine the radiative heating and cooling of the baryonic gas, while the other two simulations used tabulated heating and cooling rates and a uniform ionizing background. In order to interpret our simulation results, we develop and use an analytic model, which follows the evolution of the dynamical mass, gas, and stellar components as well as the metallicity of galaxies. We assumed an open box model with no outflows but with accretion of mass at a rate proportional to the galaxy mass, and star formation following the Kennicutt law with a critical density threshold for star formation. Our main results and conclusions can be summarized as follows.

- In all simulations, correlations between global quantities, such as the stellar massmetallicity relation, very similar to those observed for the nearby dwarf galaxies arise in simulated galaxies with stellar masses $10^{5} \mathrm{M}_{\odot} \lesssim M_{*} \lesssim 10^{8} \mathrm{M}_{\odot}$ for $z \gtrsim 3.3$.

- In galaxies of higher masses these correlations exhibit a break and the dependence of global properties on stellar mass flattens off. A similar flattening of the correlations is also found in observational data, although the location of the break may have evolved to higher masses by the present epoch (e.g., Tremonti et al. (2004)).

- The key finding of this study is that neither the inclusion of supernova energy feedback, nor the inclusion of 3-D radiative transfer significantly affects any of these correlations, a strong indication that thermal and radiative processes (as well as associated galactic outflows and winds) are not required for the observed correlations to arise. Using our analytic model, we show that these correlations in the simulated galaxies arise due the increasing inefficiency of star formation with decreasing mass of the object. The inefficiency of conversion of gas into stars and its dependence on mass are due to the critical gas column density threshold of the star formation law.

- We thus argue that trends similar to those exhibited by observed dwarf galaxies can arise without loss of gas and metals in wids, although winds may affect the exact form of the relation (normalization and slope) and its evolution.

- The observed trends of a decreasing effective yield with decreasing galaxy mass can be reproduced in our simulated galaxies reasonably well, and without winds, if the effective yield is calculated taking into account only the gas, stars, and metals that would be accessible to observations. We show that this trend is due to efficient mixing in the ISM, driven by disk instabilities, mergers, and possibly cold accretion flows. The efficient mixing redistributes the metals far beyond the stellar extent in smaller mass galaxies. Thus a significant fraction of metals in small galaxies is outside the stellar extent leading to a decrease of the estimated yield if only metals within the stellar radius are taken into account. This explanation can potentially be tested by measurements of the gas metallicity in the outskirts of the gaseous disks of dwarf galaxies.

\section{Acknowledgements}

We are grateful to Julianne Dalcanton, Josh Simon, and Francisco Prada for stimulating discussions of their results and Simon White, Volker Springel, Licia Verde, Hsiao-Wen Chen, Justin Read and Greg Bryan for helpful comments on the draft of this manuscript. This work was supported in part by the DOE and the NASA grant NAG 5-10842 at Fermilab, by the HST Theory grant HST-AR-10283.01, by the NSF grants AST-0206216, AST-0239759, and AST-0507666, and by the Kavli Institute for Cosmological Physics at 
the University of Chicago. Supercomputer simulations were run on the IBM P690 array at the National Center for Supercomputing Applications (under grant AST-020018N).

\section{References}

Arimoto, N. \& Yoshii, Y. 1987, $A \& \mathcal{E} A, 173,23$ Sharina, M. E. 2006, MNRAS, 365, 1220

Benson, A. J., Bower, R. G., Frenk, C. S., Lacey, C. G., Baugh, C. M., \& Cole, S. 2003, ApJ, 599,38

Brooks, A. M., Governato, F., Booth, C. M., Willman, B., Gardner, J. P., Wadsley, J., Stinson, G., \& Quinn, T. 2006, ApJl submitted (astro-ph/0609620)

Cole, S., Aragon-Salamanca, A., Frenk, C. S., Navarro, J. F., \& Zepf, S. E. 1994, MNRAS, 271, 781

Croton, D. J., Springel, V., White, S. D. M., De Lucia, G., Frenk, C. S., Gao, L., Jenkins, A., Kauffmann, G., Navarro, J. F., \& Yoshida, N. 2006, MNRAS, 365, 11

De Rossi, M. E., Tissera, P. B., \& Scannapieco, C. 2006, ArXiv Astrophysics e-prints

Dekel, A. \& Silk, J. 1986, ApJ, 303, 39

Dekel, A. \& Woo, J. 2003, MNRAS, 344, 1131

D'Ercole, A. \& Brighenti, F. 1999, MNRAS, 309, 941

Ferrara, A. \& Tolstoy, E. 2000, MNRAS, 313, 291

Garnett, D. R. 2002, ApJ, 581, 1019

Garnett, D. R. \& Shields, G. A. 1987, ApJ, 317, 82

Gnedin, N. Y. 2000, ApJ, 542, 535

Gnedin, N. Y. \& Abel, T. 2001, New Astronomy, 6, 437

Grebel, E. K., Gallagher, III, J. S., \& Harbeck, D. 2003, AJ, 125, 1926

Gurovich, S., McGaugh, S. S., Freeman, K. C., Jerjen, H., Staveley-Smith, L., \& De Blok, W. J. G. 2004, Publications of the Astronomical Society of Australia, 21, 412

Heckman, T. M., Lehnert, M. D., Strickland, D. K., \& Armus, L. 2000, ApJs, 129, 493

Hoeft, M., Yepes, G., Gottlöber, S., \& Springel, V. 2006, MNRAS, 371, 401

Iliev, I. T., Ciardi, B., Alvarez, M. A., \& et al. 2006, MNRAS submitted (astro-ph/0603199)

Kauffmann, G., Heckman, T. M., White, S. D. M., \& et al. 2003, MNRAS, 341, 54

Kauffmann, G., White, S. D. M., \& Guiderdoni, B. 1993, MNRAS, 264, 201

Kennicutt, R. C. 1998, ApJ, 498, 541

Kereš, D., Katz, N., Weinberg, D. H., \& Davé, R. 2005, MNRAS, 363, 2

Klypin, A., Kravtsov, A. V., Bullock, J. S., \& Primack, J. R. 2001, ApJ, 554, 903

Kobayashi, C., Springel, V., \& White, S. D. M. 2006, MNRAS submitted (astro-ph/0604107)

Kobulnicky, H. A. \& Kewley, L. J. 2004, ApJ, 617, 240

Kravtsov, A. V. 1999, Ph.D. Thesis

-. 2003, ApJl, 590, L1

Kravtsov, A. V., Gnedin, O. Y., \& Klypin, A. A. 2004, ApJ, 609, 482

Kravtsov, A. V., Klypin, A. A., \& Khokhlov, A. M. 1997, ApJs, 111, 73

Lacey, C., Guiderdoni, B., Rocca-Volmerange, B., \& Silk, J. 1993, ApJ, 402, 15

Larson, R. B. 1974, MNRAS, 169, 229

Lecavelier des Etangs, A., Désert, J.-M., Kunth, D., Vidal-Madjar, A., Callejo, G., Ferlet, R., Hébrard, G., \& Lebouteiller, V. 2004, A\& A, 413, 131

Lee, H., Skillman, E. D., Cannon, J. M., Jackson, D. C., Gehrz, R. D., Polomski, E. F., \& Woodward, C. E. 2006a, ApJ submitted (astro-ph/0605036)

Lee, H., Skillman, E. D., \& Venn, K. A. 2006b, ApJ, 642, 813

Lequeux, J., Peimbert, M., Rayo, J. F., Serrano, A., \& Torres-Peimbert, S. 1979, A\& A, 80, 155

Mac Low, M.-M. \& Ferrara, A. 1999, ApJ, 513, 142

Marcolini, A., D'Ercole, A., \& Brighenti, F. 2006, MNRAS submitted (astro-ph/0602386)

Marri, S. \& White, S. D. M. 2003, MNRAS, 345, 561

Martin, C. L. 2005, ApJ, 621, 227

Martin, C. L. \& Kennicutt, R. C. 2001, ApJ, 555, 301

Martin, C. L., Kobulnicky, H. A., \& Heckman, T. M. 2002, ApJ, 574, 663 
Mateo, M. L. 1998, ARAA, 36, 435

Matthews, L. D., van Driel, W., \& Gallagher, J. S. 1998, AJ, 116, 2196

Mayer, L., Kazantzidis, S., Mastropietro, C., \& Wadsley, J. 2007, Nature, 445, 738

McGaugh, S. S. 2005, ApJ, 632, 859

McGaugh, S. S. \& de Blok, W. J. G. 1997, ApJ, 481, 689

McGaugh, S. S., Schombert, J. M., Bothun, G. D., \& de Blok, W. J. G. 2000, ApJl, 533, L99

Miller, G. E. \& Scalo, J. M. 1979, ApJs, 41, 513

Mo, H. J., Mao, S., \& White, S. D. M. 1998, MNRAS, 295, 319

Mo, H. J., Yang, X., van den Bosch, F. C., \& Katz, N. 2005, MNRAS, 363, 1155

Navarro, J. F. \& Steinmetz, M. 1997, ApJ, 478, 13

Navarro, J. F. \& White, S. D. M. 1993, MNRAS, 265, 271

-. 1994, MNRAS, 267, 401

Ott, J., Walter, F., \& Brinks, E. 2005, MNRAS, 358, 1453

Pavlidou, V. \& Fields, B. D. 2006, ApJ in print

Penston, M. V. 1970, ApJ, 162, 771

Pettini, M., Shapley, A. E., Steidel, C. C., Cuby, J.-G., Dickinson, M., Moorwood, A. F. M., Adelberger, K. L., \& Giavalisco, M. 2001, ApJ, 554, 981

Pilyugin, L. S., Vílchez, J. M., \& Contini, T. 2004, A\&AA, 425, 849

Prada, F. \& Burkert, A. 2002, ApJl, 564, L73

Renzini, A., Ciotti, L., D'Ercole, A., \& Pellegrini, S. 1993, ApJ, 419, 52

Savaglio, S., Glazebrook, K., Le Borgne, D., \& et al. 2005, ApJ, 635, 260

Scannapieco, C., Tissera, P. B., White, S. D. M., \& Springel, V. 2006, MNRAS submitted (astro-ph/0604524)

Simon, J. D., Prada, F., Vilchez, J. M., Blitz, L., \& Robertson, B. 2006, ApJ in press (astro$\mathrm{ph} / 0606570)$

Skillman, E. D., Kennicutt, R. C., \& Hodge, P. W. 1989, ApJ, 347, 875

Slyz, A. D., Devriendt, J. E. G., Bryan, G., \& Silk, J. 2005, MNRAS, 356, 737

Somerville, R. S. \& Primack, J. R. 1999, MNRAS, 310, 1087

Springel, V. \& Hernquist, L. 2003, MNRAS, 339, 289

Strickland, D. K., Heckman, T. M., Colbert, E. J. M., Hoopes, C. G., \& Weaver, K. A. 2004, ApJ, 606, 829

Sutherland, R. S. \& Dopita, M. A. 1993, ApJs, 88, 253

Tassis, K., Abel, T., Bryan, G. L., \& Norman, M. L. 2003, ApJ, 587, 13

Tassis, K., Kravtsov, A., \& Gnedin, N. Y. 2007, ApJ, in press

Thacker, R. J. \& Couchman, H. M. P. 2001, ApJl, 555, L17

Tremonti, C. A., Heckman, T. M., Kauffmann, G., \& et al. 2004, ApJ, 613, 898

van den Bosch, F. C. 2000, ApJ, 530, 177

-. 2001, MNRAS, 327, 1334

van Zee, L. \& Haynes, M. P. 2006, ApJ, 636, 214

van Zee, L., Haynes, M. P., Salzer, J. J., \& Broeils, A. H. 1997, AJ, 113, 1618

Veilleux, S., Cecil, G., \& Bland-Hawthorn, J. 2005, ARAA, 43, 769

Verde, L., Oh, S. P., \& Jimenez, R. 2002, MNRAS, 336, 541

Verheijen, M. A. W. 2001, ApJ, 563, 694

Vitvitska, M., Klypin, A. A., Kravtsov, A. V., Wechsler, R. H., Primack, J. R., \& Bullock, J. S. 2002, ApJ, 581, 799

Warren, B. E., Jerjen, H., \& Koribalski, B. S. 2004, AJ, 128, 1152

-. 2006, AJ, 131, 2056

Wechsler, R. H., Bullock, J. S., Primack, J. R., Kravtsov, A. V., \& Dekel, A. 2002, ApJ, 568, 52

Wong, T. \& Blitz, L. 2002, ApJ, 569, 157

Woosley, S. E. \& Weaver, T. A. 1995, ApJs, 101, 181

Young, J. S., Allen, L., Kenney, J. D. P., Lesser, A., \& Rownd, B. 1996, AJ, 112, 1903

Zaritsky, D., Kennicutt, R. C., \& Huchra, J. P. 1994, ApJ, 420, 87

Zwaan, M. A., Meyer, M. J., Staveley-Smith, L., \& Webster, R. L. 2005, MNRAS, 359, L30 